\title{
Wedelolactone Acts as Proteasome Inhibitor in Breast Cancer Cells
}

\author{
Tereza Nehybová ${ }^{1,2}$, Jan Šmarda ${ }^{1}$, Lukáš Daniel ${ }^{2,3}$, Marek Stiborek ${ }^{4}$, Viktor Kanický ${ }^{4,5}$, \\ Ivan Spasojevič 6 , Jan Preisler ${ }^{4,5}$, Jiří Damborský ${ }^{2,3}$ and Petr Beneš 1,2,* \\ 1 Laboratory of Cell Differentiation, Department of Experimental Biology, Faculty of Science, \\ Masaryk University, Kamenice 5, 62500 Brno, Czech Republic; 322903@mail.muni.cz (T.N.); \\ smarda@sci.muni.cz (J.Š.) \\ 2 International Clinical Research Center, Center for Biological and Cellular Engineering, St. \\ Anne's University Hospital, Pekarska 53, 65691 Brno, Czech Republic; 211165@mail.muni.cz (L.D.); \\ jiridamborsky0@gmail.com (J.D.) \\ 3 Loschmidt Laboratories, Department of Experimental Biology and Research Centre for Toxic Compounds in \\ the Environment RECETOX, Faculty of Science, Masaryk University, Kamenice 5, 62500 Brno, Czech Republic \\ 4 Department of Chemistry, Masaryk University, Kamenice 5, 62500 Brno, Czech Republic; \\ 408516@mail.muni.cz (M.S.); viktor.kanicky@ceitec.muni.cz (V.K.); preisler@chemi.muni.cz (J.P.) \\ 5 CEITEC-Central European Institute of Technology, Masaryk University, Kamenice 5, \\ 62500 Brno, Czech Republic \\ 6 Department of Life Sciences, Institute for Multidisciplinary Research, University of Belgrade, \\ 11030 Belgrade, Serbia; redoxsci@gmail.com \\ * Correspondence: pbenes@sci.muni.cz; Tel.: +420-54949-3125; Fax: +420-54949-5533
}

Academic Editor: Toshio Morikawa

Received: 1 February 2017; Accepted: 25 March 2017; Published: 29 March 2017

\begin{abstract}
Wedelolactone is a multi-target natural plant coumestan exhibiting cytotoxicity towards cancer cells. Although several molecular targets of wedelolactone have been recognized, the molecular mechanism of its cytotoxicity has not yet been elucidated. In this study, we show that wedelolactone acts as an inhibitor of chymotrypsin-like, trypsin-like, and caspase-like activities of proteasome in breast cancer cells. The proteasome inhibitory effect of wedelolactone was documented by (i) reduced cleavage of fluorogenic proteasome substrates; (ii) accumulation of polyubiquitinated proteins and proteins with rapid turnover in tumor cells; and (iii) molecular docking of wedelolactone into the active sites of proteasome catalytic subunits. Inhibition of proteasome by wedelolactone was independent on its ability to induce reactive oxygen species production by redox cycling with copper ions, suggesting that wedelolactone acts as copper-independent proteasome inhibitor. We conclude that the cytotoxicity of wedelolactone to breast cancer cells is partially mediated by targeting proteasomal protein degradation pathway. Understanding the structural basis for inhibitory mode of wedelolactone might help to open up new avenues for design of novel compounds efficiently inhibiting cancer cells.
\end{abstract}

Keywords: breast cancer; copper; proteasome; reactive oxygen species; wedelolactone

\section{Introduction}

The ubiquitin-proteasome system (UPS) controls a highly complex and tightly regulated process of cellular protein degradation. In contrast to rather non-specific extracellular/membrane protein degradation by lysosomes, the proteasomes destroy proteins labelled with polyubiquitin chains. UPS consists of numerous protein components (E1, E2, E3 enzymes, proteasome, deubiquitinases). The mammalian cytosolic 26S proteasome complex contains the core 20S proteasome capped with one or two 195 regulatory subunit(s). The proteolytic activities are located in $\beta 1, \beta 2$, and $\beta 5$ subunits of 
the core 20S proteasome complex [1-3]. The eukaryotic proteasome possesses at least three distinct protease activities: chymotrypsin-like (cleavage after hydrophobic residues, located in $\beta 5$ subunit), trypsin-like (cleavage after basic residues, located in $\beta 2$ subunit), and caspase-like (cleavage after acidic residues, located in $\beta 1$ subunit) [4]. The chymotrypsin-like activity is usually the strongest one [5].

Deregulation of UPS has severe effect on cellular function and homeostasis. Over $80 \%$ of cellular proteins are degraded via UPS including those involved in regulation of cell proliferation, differentiation, immune signaling, and cell response to stress [3,6]. Emerging evidence show that the targeting of UPS degradation pathway might be a viable anticancer strategy. Due to increased rate of genomic mutations, transformed cells accumulate large quantities of misfolded or overexpressed proteins. In response to such accumulation, malignant cells enhance the expression and activity of UPS [5,7]. Preclinical studies have confirmed a higher susceptibility of malignant cells to cytotoxic effects of UPS inhibitors when compared to normal cells $[6,8]$. Pertinent to this, several proteasome inhibitors have entered clinical trials, and some have already been approved for the treatment of aggressive hematopoietic tumors $[9,10]$. However, tumor cell resistance and high toxicity remain an issue in solid tumors leading to search for new UPS inhibitors [5].

Wedelolactone, a natural coumestan, is one of the bioactive compounds found in extracts of Eclipta alba and Wedelia calendulacea [11]. Recently, in vitro and in vivo anti-cancer properties of wedelolactone in solid tumors including breast, colon, prostate, hepatocellular, pituitary cancers, and neuroblastoma were described in a number of reports [12-19]. Wedelolactone is clearly a multi-target compound and its anti-cancer properties were primarily attributed to the inhibition of multiple kinases, androgen receptor, 5-lipoxygenase, and the c-Myc protein [13,15,17-21]. However, it was found recently that wedelolactone also inhibits topoisomerase II $\alpha$ activity and blocks DNA synthesis in the breast cancer cells, and that these effects are promoted by copper ions, at least partially via redox interactions [12,22].

This study shows that wedelolactone acts as inhibitor of 20S/26S proteasome chymotrypsin-like and to lesser extent also trypsin-like and caspase-like activities. Treatment of breast cancer cells with wedelolactone resulted in accumulation of ubiquitinated proteins and proteins representing typical proteasomal targets, such as p21, p27, p53, and Bax. Molecular docking revealed a productive binding of wedelolactone to the active sites of $\beta 1, \beta 2$, and $\beta 5$ proteasomal subunits with a stronger preference for $\beta 5$ subunit. The proteasome inhibition by wedelolactone is not dependent on cellular copper level in breast cancer cells. This study concludes that wedelolactone acts as copper-independent inhibitor of proteasome.

\section{Results}

\subsection{Wedelolactone Inhibits Proteolytic Activities of Proteasome in Breast Cancer Cell Lines}

MDA-MB-231, MDA-MB-468, and T47D cells were exposed to increasing concentrations of wedelolactone to study its effect on proteasome in breast cancer cells. Chymotrypsin-like, trypsin-like and caspase-like activities of proteasome were evaluated in cell extracts using the activity-specific fluorogenic substrates. Wedelolactone inhibited all three proteolytic activities of proteasome with the highest potency for the chymotrypsin-like activity ( IC $_{50}$ values $27.8 \mu \mathrm{M}$ for MDA-MB-231, $12.78 \mu \mathrm{M}$ for MDA-MB-468 and $19.45 \mu \mathrm{M}$ for T47D) (Figure 1). 

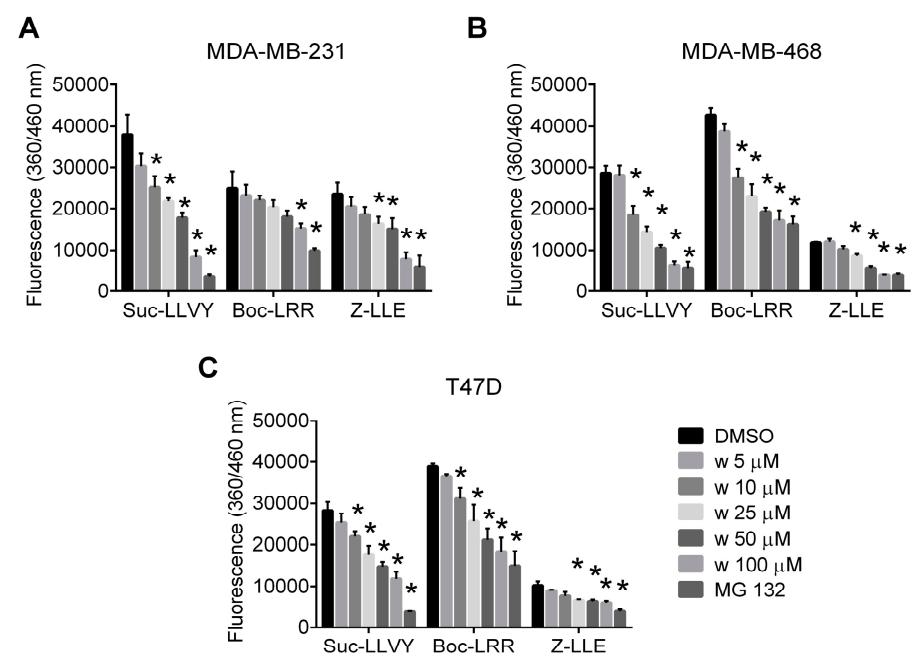

Figure 1. Wedelolactone inhibits chymotrypsin-like, trypsin-like and caspase-like activities in breast cancer cells. MDA-MB-231 (A); MDA-MB-468 (B); and T47D (C) cells were treated with various concentrations of wedelolactone $(\mathrm{w})$ for $10 \mathrm{~h}$. Proteasome activities were evaluated in cell extracts using the activity-specific fluorogenic substrates (Suc-LLVY-AMC for testing chymotrypsin-like, Z-LLE-AMC for caspase-like, and Boc-LRR-AMC for trypsin-like activities). Treatment with MG132 served as a positive control. The data represent the mean values from three independent experiments. Error bars indicate the SD. * indicates a significant $(p<0.05)$ difference between wedelolactone-/MG132- and DMSO-treated cells.

\subsection{Wedelolactone Inhibits Proteolytic Activities of Purified $20 S$ and $26 S$ Proteasome Complexes In Vitro}

The 26S proteasome purified from MDA-MB-231 cells and the commercially available $20 S$ proteasome were incubated separately with the activity-specific fluorogenic substrates and wedelolactone in various concentrations to evaluate the ability of wedelolactone to inhibit their chymotrypsin-like, trypsin-like, and caspase-like activities. Wedelolactone inhibited all three proteasomal activities in vitro in a dose-dependent manner with the highest potency against the chymotrypsin-like activity ( $\mathrm{IC}_{50}$ values $9.97 \mu \mathrm{M}$ for $26 \mathrm{~S}$ and $6.13 \mu \mathrm{M}$ for $20 \mathrm{~S}$ proteasome) (Figure 2).

A

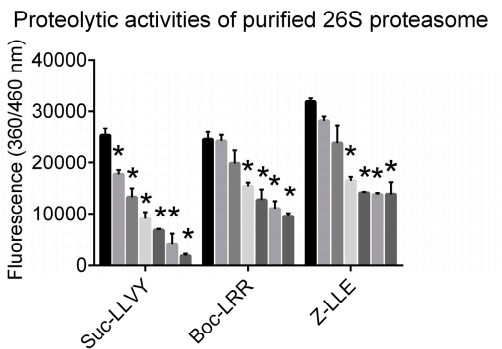

B

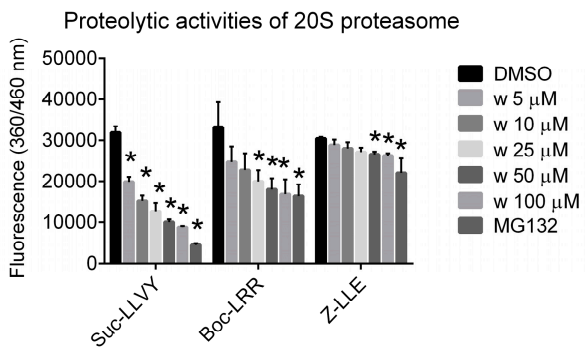

Figure 2. Wedelolactone inhibits chymotrypsin-like, trypsin-like, and caspase-like activities of purified $26 \mathrm{~S}$ and $20 \mathrm{~S}$ proteasome complexes in vitro. Wedelolactone $(\mathrm{w})$ was added at various concentrations to reaction mixture containing either (A) 26S proteasome purified from MDA-MB-231 cells or (B) commercially available $20 \mathrm{~S}$ proteasome, and fluorogenic substrate (Suc-LLVY-AMC for testing chymotrypsin-like, Z-LLE-AMC for caspase-like, and Boc-LRR-AMC for trypsin-like activities). Fluorescence was measured after $1 \mathrm{~h}$ incubation. MG132 was used as a positive control. The data represent the mean values from three independent experiments. Error bars indicate the SD. * indicates a significant $(p<0.05)$ difference in proteolytic activities between reaction mixtures containing wedelolactone/MG132 and DMSO. 


\subsection{Wedelolactone Causes Accumulation of Polyubiquitinated and Short-Lived Proteins in Breast Cancer Cells}

The level of polyubiquitinated proteins and p21, p27, p53, and Bax proteins in wedelolactone-treated MDA-MB-231, MDA-MB-468, and T47D breast cancer cells were analyzed by immunoblotting to further confirm the inhibitory effect of wedelolactone on proteasome. We found the dose-dependent accumulation of p21, p27, p53, Bax as well as multiubiquitinated proteins occurring in all three cell lines tested (Figure 3A). To confirm that the wedelolactone-induced accumulation of p21, p27, p53, and Bax proteins is not caused by increased rate of their transcription/expression, the transcripts of corresponding genes were quantified using quantitative polymerase chain reaction (qPCR). We found that wedelolactone did not affect expression of any of these genes in all three breast cancer cell lines (Figure 3B).

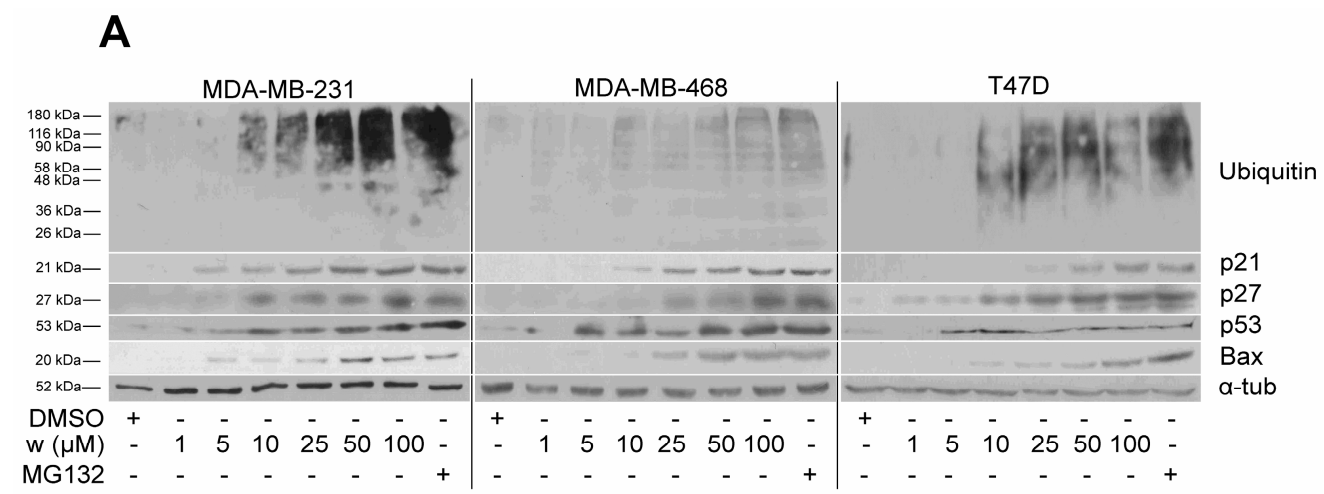

B

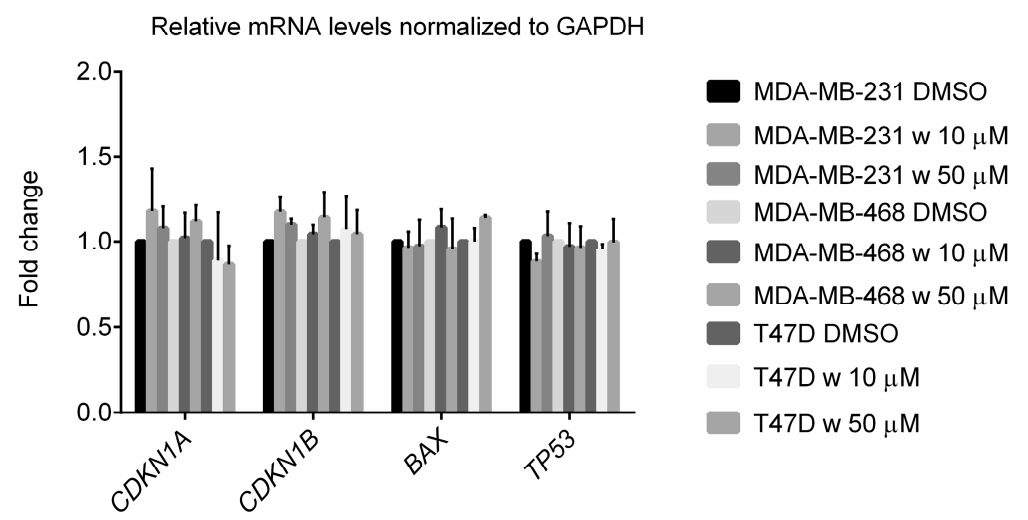

Figure 3. Multiubiquitinated and high turnover proteins accumulate in wedelolactone-treated breast cancer cells. MDA-MB-231, MDA-MB-468, and T47D cells were treated with various concentrations of wedelolactone or solvent for $10 \mathrm{~h}$. (A) Protein extracts were subsequently analyzed by SDS-PAGE and immunoblotting using p21-, p27-, p53-, Bax-, and ubiquitin-specific antibodies. Treatment with MG132 served as a positive control; (B) Transcripts of CDKN1A, CDKN1B, TP53 and BAX genes were quantified using qPCR.

\subsection{Cytotoxicity of Wedelolactone Increases for Cells with High Content of Intracellular Copper}

The authors of this study suggested previously that cytotoxicity of wedelolactone can be at least partly explained by redox-cycling with copper ions, reactive oxygen species (ROS) generation and promoted oxidative stress [22]. To confirm the role of copper ions in cytotoxicity of wedelolactone, breast cancer cells were transiently transfected with plasmid coding for human copper transporter CTR1. The transfection efficiency determined by flow-cytometry was $65.6 \% \pm 3.7 \%, 43.6 \% \pm 4.2 \%$, and $46.6 \% \pm 3.8 \%$ in MDA-MB-231, MDA-MB-468, and T47D cells, respectively (Figure S1). Expression of exogenous CTR1 protein was confirmed by immunoblotting (Figure 4A). Transfected cells were 
exposed to copper sulfate for $24 \mathrm{~h}$ or left untreated. Analysis of relative copper concentrations in cell lysates revealed that only combination of CTR1 overexpression/copper supplementation efficiently increases copper-loading of all three cell lines (Figure 4B). Therefore, for next set of experiments, copper-loaded cells were prepared by simultaneous CTR1 overexpression/copper supplementation. Copper-loaded and control cells (both over-expressing exogenous CTR1) were exposed to wedelolactone or DMSO for $48 \mathrm{~h}$ and their mortality was assessed by PI exclusion assay using flow-cytometry. In agreement with our hypothesis, cytotoxicity induced by wedelolactone was enhanced by copper loading (Figure 4C, Figure S2). Furthermore, to analyze whether copper loading enhances the wedelolactone-induced ROS production, copper-loaded and control cells (both over-expressing exogenous CTR1) were treated with wedelolactone or solvent for $10 \mathrm{~h}$ and ROS production was analyzed after DHE staining by flow-cytometry. Copper-loaded cells produced more ROS in response to wedelolactone than controls (Figure 4D, Figure S3). No significant differences in cell mortality or ROS production was observed in mock-transfected cells that were either pre-incubated with copper or left untreated and subsequently exposed to wedelolactone (Figure S4). It is hypothesized that intracellular level of copper did not reach the required threshold in this case. These results support previous findings that cytotoxicity of wedelolactone is at least partially mediated via (redox) interactions with copper ions.

A

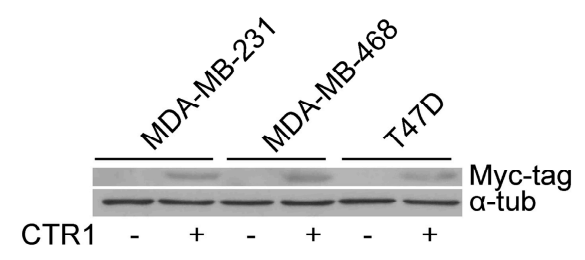

C

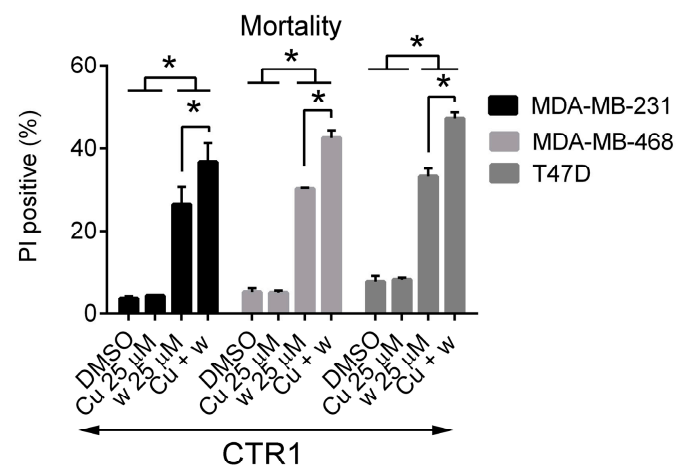

B

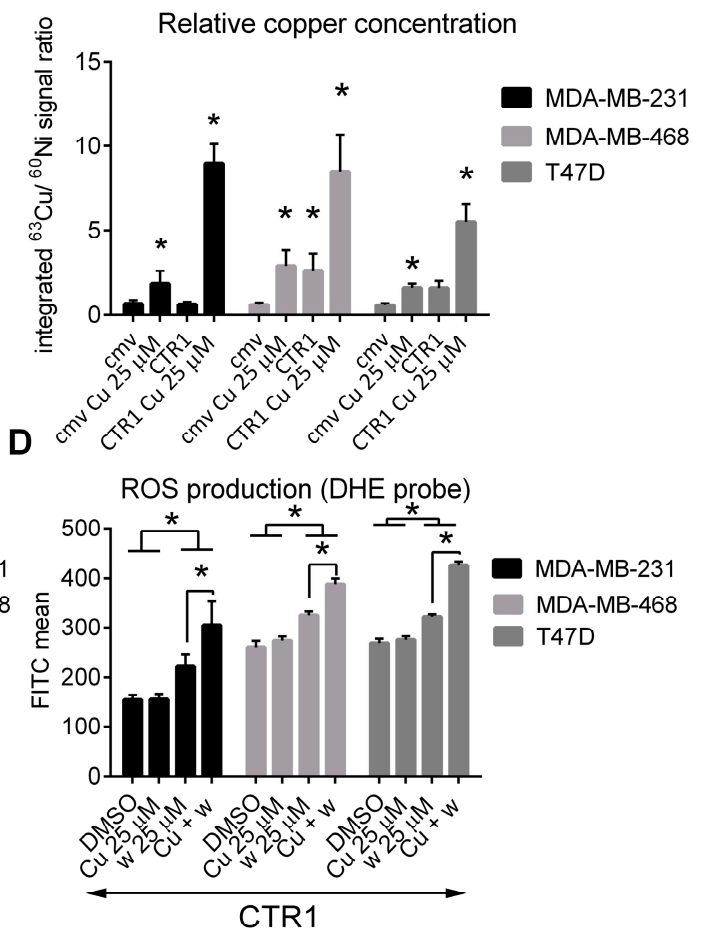

Figure 4. Copper loading enhances cytotoxicity and ROS production in wedelolactone-treated breast cancer cells. MDA-MB-231, MDA-MB-468, and T47D cells were transiently transfected with pCNDA3.1-hCTR1-N-Myc (CTR1) or control pCDNA3.1 plasmids (cmv), pretreated with copper sulfate $(\mathrm{Cu})$ for $24 \mathrm{~h}$ and subsequently treated with wedelolactone $(\mathrm{w})$ or solvent (DMSO) in fresh media. (A) Cells were harvested and expression of exogenous CTR1 protein was confirmed by SDS-PAGE followed by immunoblotting with the Myc-Tag antibody; (B) Relative copper concentration in cell lysates was analyzed by SALD ICP MS. Data for copper are presented as an integrated ${ }^{63} \mathrm{Cu} /{ }^{60} \mathrm{Ni}$ signal ratio. (C) Cell mortality and (D) ROS production were evaluated after PI/DHE staining using flow-cytometry. The data represent the mean values from three independent experiments. Error bars indicate the SD. * indicates a significant $(p<0.05)$ difference. 


\subsection{Copper Does Not Affect the Inhibition of Proteasome Activity by Wedelolactone in Breast Cancer Cell Lines}

There are several copper-interacting compounds that have been shown to inhibit proteasome $[23,24]$. To analyze whether the inhibitory effect of wedelolactone on proteolytic activities of proteasome is also mediated by copper, we compared chymotrypsin-like, trypsin-like and caspase-like proteolytic activities of proteasome in copper-loaded and control breast cancer cells (both overexpressing exogenous CTR1). The inhibition of all three proteolytic activities by wedelolactone were found to be similar in copper-loaded and control cells (Figure 5), suggesting that the inhibition of proteasome activities by wedelolactone is a copper-independent process. No significant differences in proteolytic activities were observed in mock-transfected cells that were either pre-incubated with copper or left untreated and subsequently exposed to wedelolactone (Figure S5).
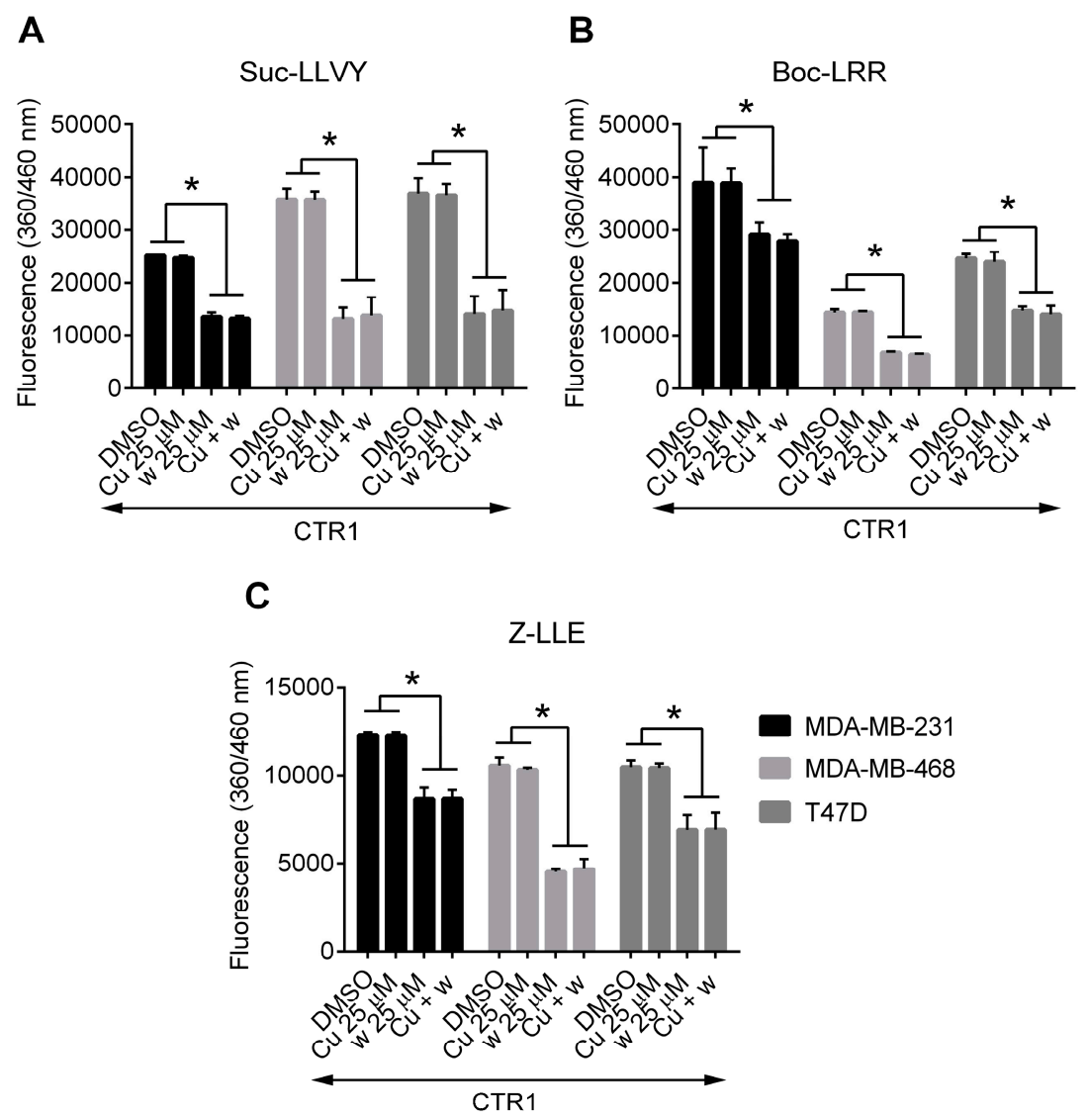

Figure 5. Copper loading does not affect inhibition of proteasome by wedelolactone. Cells were transfected with the pCNDA3.1-hCTR1-N-Myc plasmid, loaded with copper $(\mathrm{Cu})$ or left untreated and exposed to various concentrations of wedelolactone $(w)$ or solvent (DMSO) for $10 \mathrm{~h}$. Proteasome activities were evaluated in cell extracts using the activity-specific fluorogenic substrates (Suc-LLVY-AMC for testing chymotrypsin-like (A), Z-LLE-AMC for caspase-like (B), and Boc-LRR-AMC for trypsin-like activities (C)). The data represent the mean values from three independent experiments. Error bars indicate the SD. * indicates a significant $(p<0.05)$ difference.

\subsection{Molecular Docking of Wedelolactone to the Active Sites of Proteasome}

To reveal the mechanism of proteasome inhibition by wedelolactone, in silico docking analysis was performed. Since the functional units of proteasome are located at each of the inner $\beta$ rings, the blind docking was initially performed to assess the specificity of wedelolactone to the active sites. This analysis revealed that wedelolactone occupied the active site of the $\beta 5$ subunit at least three times more often than the active sites in other units of the protein. 
The focused docking identified similar binding mode of wedelolactone in the $\beta 1$ and $\beta 2$ subunits. Aside from the hydrophobic contacts, wedelolactone formed specific H-bond with the backbone of Thr21 and Gly47 in both subunits. Moreover, wedelolactone formed an additional H-bond with the side-chain of Thr20 in the $\beta 1$ subunit. Wedelolactone sterically blocked the catalytic residue Thr1 in both $\beta 1$ and $\beta 2$ subunits, possibly modulating its proteolytic activity (Figure 6A,B). The focused docking identified a different binding mode of wedelolactone in the $\beta 5$ subunit. Aside from the specific H-bond with the backbone of Gly47, two hydroxyl groups of wedelolactone were able to form H-bond with the catalytic residue Thr1 (Figure 6C). This specific interaction might be responsible for the preferred binding to the $\beta 5$ active site over the $\beta 1$ and $\beta 2$ active sites, observed in the blind docking. Beside the steric hindrance, the interaction with the catalytic residue Thr1 might be responsible for the elevated inhibition of chymotrypsin-like activity observed experimentally.

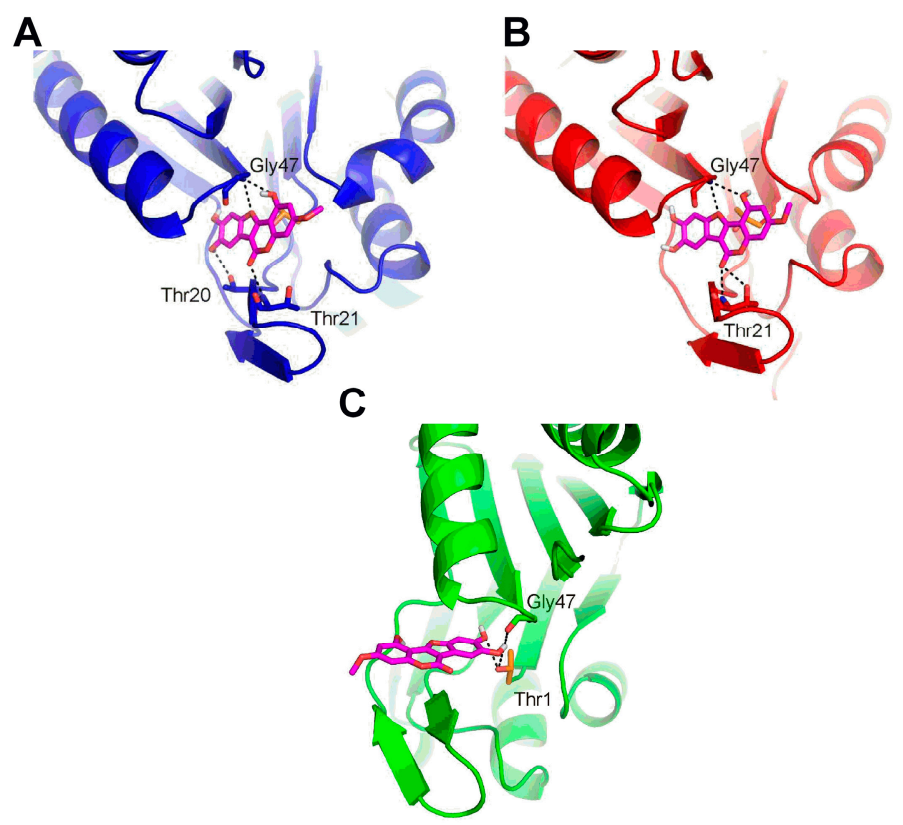

Figure 6. The binding modes of wedelolactone in the $\beta 1(\mathbf{A}), \beta 2(\mathbf{B})$, and $\beta 5(\mathbf{C})$ subunits of the yeast $20 \mathrm{~S}$ proteasome. Wedelolactone is represented as magenta sticks, $\beta 1, \beta 2$, and $\beta 5$ subunits are represented as blue, red, and green, respectively. Dashed lines represent the specific H-bonds to the active sites residues. The catalytic residue Thr1 is represented as orange sticks.

\section{Discussion}

Wedelolactone is a natural polyphenolic catechol-type compound with anti-cancer effects that are exerted via multiple mechanisms/targets [12-21]. In our previous studies, we reported that cytotoxic effect of wedelolactone can be partially attributed to its pro-oxidative and DNA damage activity that is promoted by copper ions. Such activities most likely involve production of ROS and (semi)quinones [22] and were previously described for other polyphenolic compounds [25-27]. Very recently, copper-mediated cytotoxicity was confirmed also for coumestrol, another coumestan with structure similar to that of wedelolactone [28]. In accordance with this, cytotoxicity of wedelolactone was enhanced here by copper loading in three breast cancer cell lines. Previously, quinones and ROS, formed by oxidative metabolism of catechol-type polyphenol dopamine, were reported to act as proteasome inhibitors [29]. We found that copper overloading significantly enhanced wedelolactone-induced ROS production and cytotoxicity but it did not further enhance its proteasome inhibitory properties, suggesting the copper-independent mechanism of proteasome inhibition by wedelolactone. 
The structural basis of proteasome inhibition by wedelolactone was subsequently revealed by molecular docking. Molecular structure of the core $20 \mathrm{~S}$ proteasome is extremely conserved and is organized in four stacked rings, each formed by seven subunits in an $\alpha 7 \beta 7 \beta 7 \alpha 7$ configuration. Seven distinct $\beta$ subunits are carrying the enzyme active sites, specifically $\beta 1$ carries caspase-like activity, $\beta 2$ is responsible for trypsin-like activity and $\beta 5$ encodes chymotrypsin-like activity [30]. Protein degradation is facilitated by nucleophilic N-terminal threonine (Thr1) residues of catalytic $\beta$ subunits, in which the side chain hydroxyl group reacts with peptide bonds of substrates as well as functional groups of inhibitors [31]. Inhibitors of the $20 \mathrm{~S}$ proteasome can be divided into two main groups based on whether or not they form a covalent bond with the active site Thr1 according to classification proposed by Kisselev et al. [10]. Molecular docking revealed that wedelolactone occupies the active sites of $\beta 1, \beta 2$, and $\beta 5$ proteasomal subunits. While similar binding mode was predicted for $\beta 1$ and $\beta 2$ subunit, a specific interaction between both hydroxyl groups of wedelolactone with catalytic residue Thr1 was observed only in $\beta 5$ subunit. These differences in binding modes are probably responsible also for predicted favored interaction with $\beta 5$ subunit and might explain a stronger inhibition of proteasomal chymotrypsin-like activity compared to trypsin- and caspase-like activities.

The observed $\mathrm{IC}_{50}$ values for chymotrypsin-like inhibitory activity of wedelolactone were below $10 \mu \mathrm{M}$ in vitro and within $10-25 \mu \mathrm{M}$ range in cells. It is noteworthy that wedelolactone induced growth arrest and apoptosis in all three breast cancer cell line tested at concentrations corresponding to the above mentioned $\mathrm{IC}_{50}$ values [12,22]. This suggests that inhibition of proteasome may contribute significantly to cytotoxicity of this compound.

Inhibition of proteasome results in increased levels of polyubiquitinated proteins because most of the proteasome-mediated protein degradation pathways require ubiquitination [32]. Moreover proteins with high turnover, including p21, p27, p53, and Bax accumulates in cells in response to proteasomal inhibition [33-35]. Such accumulation was clearly documented here in wedelolactone-treated cells. It is important to note that some previous studies showing connection between the treatment with wedelolactone and altered expression of numerous proteins should be interpreted with caution as wedelolactone can affect not only protein expression but also protein degradation pathway.

This study concluded that natural coumestan wedelolactone acts as a copper-independent proteasome inhibitor with potency similar to other flavonoids. As cancer cells were reported to be more sensitive to proteasome inhibition, this novel function of wedelolactone might explain its preferred toxicity towards cancer cells observed previously [20]. Understanding a structural basis for inhibitory mode of wedelolactone might help to open up new avenues for design of novel compounds efficiently inhibiting cancer cells.

\section{Material and Methods}

\subsection{Chemicals and Plasmids}

Chemicals were obtained from commercial providers: wedelolactone, dimethyl sulfoxide (DMSO), propidium iodide (PI), and copper sulfate (Sigma-Aldrich, St. Louis, MO, USA), dihydroethidium (DHE; Cayman Pharma, Ann Arbor, MI, USA), Proteasome Activity Fluorometric Assay Kit II (UPBio, Aurora, CO, USA). The pCNDA3.1-hCTR1-N-Myc plasmid was kindly provided by Dennis J. Thiele [36].

\subsection{Cell Culture}

The human breast cancer cell lines MDA-MB-231, MDA-MB-468, and T47D were cultured in HEPES-modified RPMI 1640 medium (Sigma-Aldrich) supplemented with 10\% fetal calf serum (FCS, Sigma-Aldrich), $2 \mathrm{mM}$ L-glutamine, $100 \mathrm{U} / \mathrm{mL}$ penicillin, and $100 \mu \mathrm{g} / \mathrm{mL}$ streptomycin (Lonza, Verviers, Belgium) in a humidified atmosphere of $5 \% \mathrm{CO}_{2}$ at $37^{\circ} \mathrm{C}$. In all experiments, wedelolactone was applied at concentrations that have been shown previously to effectively induce cell death in breast cancer cell lines [12,22]. 


\subsection{Proteasome Activity Assay}

\subsubsection{Purification of 26S Proteasome from MDA-MB-231 Cells}

Human 26S proteasome was purified from $8 \times 10^{6}$ of MDA-MB-231 cells using The Rapid 26S Proteasome Purification Kit (J4310, UBPBio) according to manufacturer's instructions.

\subsubsection{Proteasome Activity In Vitro}

Chymotrypsin-like, trypsin-like and caspase-like proteasome activities were determined using Proteasome Activity Fluorometric Assay Kit II (J4120, UBPBio) according to manufacturer's instructions. Briefly, wedelolactone was added at various concentrations to $150 \mu \mathrm{L}$ reaction mixture containing either purified $10 \mathrm{nM}$ bovine $20 \mathrm{~S}$ proteasome (A1400, UBPBio) or $5 \mu \mathrm{g}$ of MDA-MB-231-purified 26S proteasome complex, and $50 \mu \mathrm{M}$ fluorogenic substrate (Suc-LLVY-AMC to test chymotrypsin-like activity, Z-LLE-AMC to test caspase-like activity and Boc-LRR-AMC to test trypsin-like activity) in $1 \times$ Proteasome Assay Buffer (40 mM Tris, pH 7.1, 2 mM $\beta$-mercaptoethanol; UBPBio). MG132 at concentration of $10 \mu \mathrm{M}$ and aliquots of DMSO were used as positive and negative controls, respectively. Fluorescence was measured by TECAN infinite 200 plate reader (TECAN, Mannedorf, Switzerland) for $1 \mathrm{~h}$ at $37^{\circ} \mathrm{C}$.

\subsubsection{Proteasome Activity in Cancer Cell Lines}

MDA-MB-231, MDA-MB-468 and T47D $\left(6 \times 10^{5}\right)$ cells were seeded in $5 \mathrm{~mL}$ of culture media, exposed to various concentrations of wedelolactone, DMSO or $10 \mu \mathrm{M}$ MG132 for $10 \mathrm{~h}$. Cells were harvested, resuspended in cell lysis buffer (40 mM Tris, pH 7.2, $50 \mathrm{mM} \mathrm{NaCl}, 2 \mathrm{mM} \beta$-mercaptoethanol, $2 \mathrm{mM}$ ATP, $5 \mathrm{mM} \mathrm{MgCl}, 10 \%$ Glycerol) and briefly sonicated using an Ultrasonic Processor UP100H (Hielscher, Ringwood, NJ, USA). Cell lysates were cleared by centrifugation and protein concentration in supernatant was determined using DC protein assay (Biorad, Hercules, CA, USA). Protein extract $(50 \mu \mathrm{g})$ was mixed with $50 \mu \mathrm{M}$ of fluorogenic substrates (UBPBio) in $1 \times$ Proteasome Assay Buffer in a total volume of $100 \mu \mathrm{L}$. Fluorescence was measured by TECAN infinite 200 plate reader (TECAN) for $1 \mathrm{~h}$ at $37^{\circ} \mathrm{C}$.

\subsubsection{Proteasome Activity after Copper-Overloading}

MDA-MB-231, MDA-MB-468, and T47D cells $\left(6 \times 10^{5}\right)$ were seeded in $5 \mathrm{~mL}$ of culture media. Next day, transient transfection was performed, using $4 \mu \mathrm{L}$ of Lipofectamine LTX reagent (Invitrogen, Carlsbad, CA, USA) with a mixture containing $2 \mu \mathrm{g}$ of pCDNA3.1-hCTR1-N-Myc or control pCDNA3.1 plasmid and $2 \mu \mathrm{L}$ of PLUS reagent (Invitrogen). Six hours later, the medium was replaced, cells were treated with $25 \mu \mathrm{M}$ copper sulfate or left untreated for $24 \mathrm{~h}$, and then were exposed to wedelolactone or DMSO for $10 \mathrm{~h}$ in fresh media. Cells were then harvested and proteasome activity was analyzed as described in 4.3.3.

\subsection{Immunoblotting}

$5 \times 10^{5}$ cells were seeded in 6-well plates. The next day, the cells were exposed to various concentrations of wedelolactone, DMSO, or $10 \mu \mathrm{M}$ MG132 for $10 \mathrm{~h}$. Cells were harvested and lysed as described previously [37]. Cell lysates were subjected to SDS-PAGE and immunoblotted. Sample loading was normalized according to protein concentration determined by DC protein assay (Biorad). Blots were probed with anti-ubiquitin (3933S; Cell Signaling Technology, Inc., Beverly, MA, USA), anti-p21, anti-p27 (sc-817 and sc-528; Santa Cruz Biotechnology Inc., Santa Cruz, CA, USA), anti-p53, anti-Bax or anti-Myc-Tag (9282, 5023 and 2276S; Cell Signaling Technology), anti- $\alpha$-tubulin antibodies (T9026; Sigma-Aldrich), and secondary antibodies conjugated with peroxidase (Sigma-Aldrich). Blots were developed with a standard ECL procedure with Immobilon Western Chemiluminiscent HRP Substrate (Millipore, Billerica, MA, USA). 


\subsection{RNA Isolation, $c D N A$ Synthesis and $q P C R$}

$1 \times 10^{6}$ cells were seeded in $5 \mathrm{~mL}$ dishes. Next day, the cells were exposed to various concentrations of wedelolactone or DMSO for $10 \mathrm{~h}$. Cells were harvested and total RNA was isolated using GenElute Mammalian Total RNA Miniprep kit (Sigma-Aldrich). For cDNA synthesis, $1 \mu \mathrm{g}$ of total RNA was reverse-transcribed using the QuantiTect Reverse Transcription kit (Qiagen, Hilden, Germany) according to the manufacturer's instructions in a final reaction volume of $20 \mu \mathrm{L}$. Expression of CDKN1A, CDKN1B, TP53 and BAX genes was determined using the target-specific primers (Table S1) and KAPA SYBR FAST qPCR MASTER MIX (KK460, Kapa Biosystems, Cambridge, MA, USA) on LightCycler 480 II (Roche, Basel, Switzerland). Expression of the reference GAPDH gene (probe 4326317E, ThermoFisher Scientific, Waltham, MA, USA) was used for data normalization.

\subsection{Cell Mortality}

$3 \times 10^{5}$ cells were seeded in 6-well plates. After $24 \mathrm{~h}$, transient transfection was performed, using $3 \mu \mathrm{L}$ of Lipofectamine LTX reagent (Invitrogen) with a mixture containing $1.5 \mu \mathrm{g}$ of pCNDA3.1-hCTR1-N-Myc or control pCDNA3.1 plasmid and $1.5 \mu \mathrm{L}$ of PLUS reagent (Invitrogen). Six hours later, the medium was replaced, cells were either exposed to $25 \mu \mathrm{M}$ copper sulfate for $24 \mathrm{~h}$ or left untreated and subsequently subjected to wedelolactone or DMSO for $48 \mathrm{~h}$ in fresh media. Cytotoxicity of wedelolactone was analyzed $48 \mathrm{~h}$ later by PI staining $(1 \mu \mathrm{g} / \mathrm{mL})$ using flow-cytometry as described previously [38].

\subsection{Reactive Oxygen Species Production Analysis}

$3 \times 10^{5}$ cells were seeded in 6-well plates. After $24 \mathrm{~h}$, transient transfection was performed as described in chapter 4.6. Medium was replaced after $6 \mathrm{~h}$, cells were left untreated or pretreated with $25 \mu \mathrm{M}$ copper sulfate for $24 \mathrm{~h}$, and subsequently exposed to wedelolactone or DMSO for $10 \mathrm{~h}$ in fresh media. The cells were washed with PBS and stained with $10 \mu \mathrm{M}$ DHE for $20 \mathrm{~min}$ at $37^{\circ} \mathrm{C}$ in the dark. Reactive oxygen species (ROS) were measured using flow-cytometry (BD FACSVerse, BD Biosciences, Franklin Lakes, NJ, USA) at an excitation wavelength of $485 \mathrm{~nm}$ and an emission wavelength of $538 \mathrm{~nm}$. Data were analyzed using BD FACSuite software (BD Biosciences).

\subsection{Analysis of Copper Concentrations in Cells}

For detection of intracellular copper concentrations, $6 \times 10^{5}$ cells were seeded in $5 \mathrm{~mL}$ of growth medium. The next day, the cells were transiently transfected with CTR1 or control pCDNA3.1 vector. Transfection was performed using $4 \mu \mathrm{L}$ of the Lipofectamine LTX reagent (Invitrogen) with a mixture containing $2 \mu \mathrm{g}$ of plasmid and $2 \mu \mathrm{L}$ of PLUS reagent (Invitrogen). Six hours later, the medium was replaced with fresh one and cells were pretreated with $25 \mu \mathrm{M}$ copper sulfate or left untreated for $24 \mathrm{~h}$. Then, the cells were exposed to $25 \mu \mathrm{M}$ wedelolactone or DMSO for $10 \mathrm{~h}$ in fresh media. Cells were harvested and cell lysates prepared as described previously [39]. Briefly, pelleted cells were washed twice with $1 \times$ PBS and $1.0 \times 10^{6}$ cell were lysed in a mixture of $3 \mathrm{M} \mathrm{HCl} / 10 \%$ trichloroacetic acid at room temperature for $3 \mathrm{~h}$ followed by incubation at $70{ }^{\circ} \mathrm{C}$ for $5 \mathrm{~h}$. The lysate was centrifuged (600 g/5 min) to remove cell debris and the total amount of copper in supernatant was determined by substrate-assisted laser desorption inductively-coupled plasma mass spectrometry (SALD ICP MS) [40].

Each sample was mixed with $400 \mu \mathrm{g} / \mathrm{L}$ aqueous solution containing nickel as an internal standard (ASTASOL- ${ }^{\circledR} \mathrm{Ni}, \mathrm{CRM}$, ANALYTIKA ${ }^{\circledR}$, Prague, Czech Republic) in the 1:1 ratio and spotted by a micropipette onto a polyethylene terephthalate plate (PET) as a $200 \mathrm{~nL}$ droplet in seven replicates. The sample plate was inserted into an ablation system (model UP 213, New Wave, Fremont, CA, USA) and spots were scanned by an Nd:YAG $213 \mathrm{~nm}$ laser beam in a zig-zag shaped raster with the raster spacing $190 \mu \mathrm{m}$; the laser beam waist was adjusted to the size $\sim 250 \mu \mathrm{m}$. Size of the raster was selected according to the spot diameter to desorb the entire sample (typical spot diameter $\sim 1.4 \mathrm{~mm}$ ), and the analysis time of each sample was approximately $2 \mathrm{~min}$. The ablation cell was flushed with a carrier 
gas (helium, flow rate $1.0 \mathrm{~L} / \mathrm{min}$ ), which transported the aerosol to an ICP mass spectrometer (model 7500CE, Agilent Technologies, Santa Clara, CA, USA). A sample gas flow of argon was admixed to the helium carrier gas flow subsequent to the laser ablation cell $(0.6 \mathrm{~L} / \mathrm{min})$. Optimization of LA ICP MS conditions (gas flow rates, sampling depth, electrostatic lens voltages of the MS) was performed with the glass reference material NIST SRM 612 regarding the maximum signal-to-noise ratio and minimum oxide formation $\left(\mathrm{ThO}^{+} / \mathrm{Th}^{+}\right.$counts ratio $0.2 \%, \mathrm{U}^{+} / \mathrm{Th}^{+}$counts ratio $1.1 \%$ ). Other ICP MS parameters were adjusted in compliance with the manufacturer's recommendations. The laser fluence was $\sim 0.75 \mathrm{~J} / \mathrm{cm}^{2}$, the repetition rate $10 \mathrm{~Hz}$, and the scan rate $160 \mu \mathrm{m} / \mathrm{s}$. The ions were measured with an integration time $0.1 \mathrm{~s}$. Both the flush time and the laser warm-up time were set to $10 \mathrm{~s}$. The ion signal of two copper isotopes, ${ }^{63} \mathrm{Cu}$ and ${ }^{65} \mathrm{Cu}$, was monitored to reveal possible polyatomic interferences. The signal ratio of the most abundant isotopes, ${ }^{63} \mathrm{Cu}$ and ${ }^{60} \mathrm{Ni}$ as the internal standard was used for data evaluation.

\subsection{Molecular Docking}

The three-dimensional structure of wedelolactone was downloaded from ZINC database [41], (ZINC ID: ZINC6483512). The output file in Sybyl mol2 format was converted into AutoDock Vina [42] compliant pdbqt format by MGLTools [43]. The crystal structure of yeast 20S proteasome (PDB ID: $5 \mathrm{CZ4})$ was used as a target in molecular docking. All ligands and water molecules were removed from the target molecule. The hydrogen atoms were added to the target by PyMol [44]. The Gasteiger charges and AutoDock atom types were assigned to targets by MGLTools. The active site of $\beta 1$ (caspase-like activity), $\beta 2$ (trypsin-like activity) and $\beta 5$ (chymotrypsin-like activity) subunits and both inner $\beta$ rings were selected as target regions for molecular docking performed by AutoDock Vina. The region selected for focused docking was represented by a box of $22.5 \AA \times 22.5 \AA \times 22.5 \AA$ centered at the catalytic residue Thr1. The entire protein surface was selected for a blind docking to assess the specificity of wedelolactone towards the enzyme active sites. The region selected for the blind docking was represented by a box with $87.5 \AA \times 87.5 \AA \times 87.5 \AA$ dimension centered at the middle of the two inner $\beta$ rings harboring the active sites. Ten and twenty conformations were produced by AutoDock Vina in the focused and blind docking, respectively. The docked conformations were re-scored by NNScore 2.0 [45], which predicts binding affinity of the conformation as an average over 20 distinct neural-networks.

\subsection{Statistics}

Values were expressed as means \pm standard deviations (SD). To determine statistical significance, the values were compared using a two-tailed $t$-test for unpaired samples. Differences were considered to be statistically significant with the $p$-value $<0.05$. $\mathrm{IC}_{50}$ values were determined by nonlinear regression using GraphPad PRISM 6 software (GraphPad-San Diego, CA, USA). All results were reproduced at least in three independent experiments.

Supplementary Materials: Supplementary materials can be found at www.mdpi.com/1422-0067/18/4/729/s1.

Acknowledgments: This work was funded by the projects No. LQ1601, LQ1605 and LO1214 from the National Program of Sustainability II (MEYS CR), 15-05387S from the Czech Science Foundation, and MUNI/A/0967/2015 from Masaryk University. We thank Lucia Knopfova for help with manuscript preparation. The funders had no role in study design, data collection and analysis, decision to publish, or preparation of the manuscript.

Author Contributions: Tereza Nehybová, Petr Beneš, and Ivan Spasojevič conceived the project; Tereza Nehybová, Marek Stiborek, and Lukáš Daniel performed the experiments; Tereza Nehybová, Petr Beneš, Lukáš Daniel, Marek Stiborek, Jiří Damborský, and Jan Preisler analyzed the data; Viktor Kanický and Jan Šmarda supported the project with experimental techniques; Tereza Nehybová, Petr Beneš, Ivan Spasojevič, Jiří Damborský, Jan Šmarda, and Jan Preisler wrote and revised the manuscript; Petr Beneš, Jan Šmarda, Jan Preisler, and Jiří Damborský supervised the study. All authors read and approved the final manuscript.

Conflicts of Interest: The authors declare no conflict of interest. 


\section{Abbreviations}

$\begin{array}{ll}\text { DHE } & \text { Dihydroethidium } \\ \text { DMSO } & \text { Dimethyl sulfoxide } \\ \text { ECL } & \text { Enhanced chemiluminescence } \\ \text { FCS } & \text { Fetal calf serum } \\ \text { CTR1 } & \text { Human copper transporter 1 } \\ \text { PDB } & \text { Protein data bank } \\ \text { PI } & \text { Propidium iodide } \\ \text { ROS } & \text { Reactive oxygen species } \\ \text { SALD ICP MS } & \text { Substrate-assisted laser desorption inductively-coupled plasma mass spectrometry } \\ \text { SDS-PAGE } & \text { Sodium dodecyl sulfate polyacrylamide gel electrophoresis } \\ \text { qPCR } & \text { Quantitative polymerase chain reaction } \\ \text { UPS } & \text { Ubiquitin-proteasome system }\end{array}$

\section{References}

1. Groll, M.; Heinemeyer, W.; Jäger, S.; Ullrich, T.; Bochtler, M.; Wolf, D.H.; Huber, R. The catalytic sites of $20 \mathrm{~S}$ proteasomes and their role in subunit maturation: A mutational and crystallographic study. Proc. Natl. Acad. Sci. USA 1999, 96, 10976-10983. [CrossRef] [PubMed]

2. Heinemeyer, W.; Fischer, M.; Krimmer, T.; Stachon, U.; Wolf, D.H. The active sites of the eukaryotic $20 \mathrm{~S}$ proteasome and their involvement in subunit precursor processing. J. Biol. Chem. 1997, 272, 25200-25209. [CrossRef] [PubMed]

3. Shen, M.; Schmitt, S.; Buac, D.; Duo, Q.P. Targeting the ubiquitin-proteasome system for cancer therapy. Expert Opin. Ther. Targets 2013, 17, 1091-1108. [CrossRef] [PubMed]

4. Groll, M.; Huber, R. Purification, crystallization, and X-ray analysis of the yeast $20 \mathrm{~S}$ proteasome. Methods Enzymol. 2005, 398, 329-336. [PubMed]

5. Grigoreva, T.A.; Tribulovich, V.G.; Garabadzhiu, A.V.; Melino, G.; Barlev, N.A. The 26S proteasome is a multifaceted target for anti-cancer therapies. Oncotarget 2015, 6, 24733-24749. [CrossRef] [PubMed]

6. Crawford, L.J.; Walker, B.; Irvine, A.E. Proteasome inhibitors in cancer therapy. J. Cell Commun. Signal. 2011, 5, 101-110. [PubMed]

7. Arlt, A.; Bauer, I.; Schafmayer, C.; Tepel, J.; Müerköster, S.S.; Brosch, M.; Röder, C.; Kalthoff, H.; Hampe, J.; Moyer, M.P.; et al. Increased proteasome subunit protein expression and proteasome activity in colon cancer relate to an enhanced activation of nuclear factor E2-related factor 2 (Nrf2). Oncogene 2009, 28, 3983-3996. [CrossRef] [PubMed]

8. Rajkumar, S.V.; Richardson, P.G.; Hideshima, T.; Anderson, K.C. Proteasome Inhibition As a Novel Therapeutic Target in Human Cancer. J. Cell. Oncol. 2005, 23, 630-639. [CrossRef] [PubMed]

9. Kubiczkova, L.; Pour, L.; Sedlarikova, L.; Hajek, R.; Sevcikova, S. Proteasome inhibitors-Molecular basis and current perspectives in multiple myeloma. J. Cell. Mol. Med. 2014, 18, 947-961. [CrossRef] [PubMed]

10. Kisselev, A.F.; van der Linden, W.A.; Overkleeft, H.S. Proteasome inhibitors: An expanding army attacking a unique target. Chem. Biol. 2012, 19, 99-115. [CrossRef] [PubMed]

11. Wagner, H.; Geyer, B.; Kiso, Y.; Hikino, H.; Rao, G.S. Coumestans as the main active principles of the liver drugs Eclipta alba and Wedelia calendulacea. Planta Med. 1986, 5, 370-374. [CrossRef]

12. Benes, P.; Knopfova, L.; Trcka, F.; Nemajerova, A.; Pinheiro, D.; Soucek, K.; Fojta, M.; Smarda, J. Inhibition of topoisomerase II $\alpha$ : Novel function of wedelolactone. Cancer Lett. 2011, 303, 29-38. [CrossRef] [PubMed]

13. Chen, Z.; Sun, X.; Shen, S.; Zhang, H.; Ma, X.; Liu, J.; Kuang, S.; Yu, Q. Wedelolactone a naturally occurring coumestan, enhances interferon-G signaling through inhibiting STAT1 protein dephosphorylation. J. Biol. Chem. 2013, 288, 14417-14427. [CrossRef] [PubMed]

14. Idris, A.I.; Libouban, H.; Nyangoga, H.; Landao-Bassonga, E.; Chappard, D.; Ralston, S.H. Pharmacologic inhibitors of IkappaB kinase suppress growth and migration of mammary carcinosarcoma cells in vitro and prevent osteolytic bone metastasis in vivo. Mol. Cancer Ther. 2009, 8, 2339-2347. [CrossRef] [PubMed]

15. Lin, F.M.; Chen, L.R.; Lin, E.H.; Ke, F.C.; Chen, H.Y.; Tsai, M.J.; Hsiao, P.W. Compounds from Wedelia chinensis synergistically suppress androgen activity and growth in prostate cancer cells. Carcinogenesis 2007, 28, 2521-2529. [CrossRef] [PubMed] 
16. Nehybova, T.; Smarda, J.; Benes, P. Plant coumestans: Recent advances and future perspectives in cancer therapy. Anticancer Agents Med. Chem. 2014, 14, 1351-1362. [CrossRef] [PubMed]

17. Sukumari-Ramesh, S.; Bentley, J.N.; Laird, M.D.; Singh, N.; Vender, J.R.; Dhandapani, K.M. Dietary phytochemicals induce p53- and caspase-independent cell death in human neuroblastoma cells. Int. J. Dev. Neurosci. 2011, 29, 701-710. [CrossRef] [PubMed]

18. Tsai, C.H.; Lin, F.M.; Yang, Y.C.; Lee, M.T.; Cha, T.L.; Wu, G.J.; Hsieh, S.C.; Hsiao, P.W. Herbal extract of Wedelia chinensis attenuates androgen receptor activity and orthotopic growth of prostate cancer in nude mice. Clin. Cancer Res. 2009, 15, 5435-5444. [CrossRef] [PubMed]

19. Vender, J.R.; Laird, M.D.; Dhandapani, K.M. Inhibition of NFkB reduces cellular viability in GH3 pituitary adenoma cells. Neurosurgery 2008, 62, 1122-1127. [CrossRef] [PubMed]

20. Sarveswaran, S.; Gautam, S.C.; Ghosh, J. Wedelolactone a medicinal plant-derived coumestan, induces caspase-dependent apoptosis in prostate cancer cells via downregulation of PKCe without inhibiting Akt. Int. J. Oncol. 2012, 41, 2191-2199. [PubMed]

21. Sarweswaran, S.; Ghosh, R.; Parikh, R.; Ghosh, J. Wedelolactone, an Anti-inflammatory Botanical, Interrupts c-Myc Oncogenic Signaling and Synergizes with Enzalutamide to Induce Apoptosis in Prostate Cancer Cells. Mol. Cancer Ther. 2016, 15, 2791-2801. [CrossRef] [PubMed]

22. Benes, P.; Alexova, P.; Knopfova, L.; Spanova, A.; Smarda, J. Redox state alters anti-cancer effects of wedelolactone. Environ. Mol. Mutagen. 2012, 53, 515-524. [CrossRef] [PubMed]

23. Daniel, K.G.; Gupta, P.; Harbach, R.H.; Guida, W.C.; Dou, Q.P. Organic copper complexes as a new class of proteasome inhibitors and apoptosis inducers in human cancer cells. Biochem. Pharmacol. 2004, 67, 1139-1151. [CrossRef] [PubMed]

24. Ding, W.Q.; Liu, B.; Vaught, J.L.; Yamauchi, H.; Lind, S.E. Anticancer activity of the antibiotic clioquinol. Cancer Res. 2005, 65, 3389-3395. [PubMed]

25. Arif, H.; Rehmani, N.; Farhan, M.; Ahmad, A.; Hadi, S.M. Mobilization of Copper ions by Flavonoids in Human Peripheral Lymphocytes Leads to Oxidative DNA Breakage: A Structure Activity Study. Int. J. Mol. Sci. 2015, 16, 26754-26769. [CrossRef] [PubMed]

26. Farhan, M.; Khan, H.Y.; Oves, M.; Al-Harrasi, A.; Rehmani, N.; Arif, H.; Hadi, S.M.; Ahmad, A. Cancer Therapy by Catechins Involves Redox Cycling of Copper Ions and Generation of Reactive Oxygen species. Toxins 2016, 8, 37. [CrossRef] [PubMed]

27. Khan, H.Y.; Zubair, H.; Faisal, M.; Ullah, M.F.; Farhan, M.; Sarkar, F.H.; Ahmad, A.; Hadi, S.M. Plant polyphenol induced cell death in human cancer cells involves mobilization of intracellular copper ions and reactive oxygen species generation: A mechanism for cancer chemopreventive action. Mol. Nutr. Food Res. 2014, 58, 437-446. [CrossRef] [PubMed]

28. Zafar, A.; Singh, S.; Naseem, I. Cytotoxic activity of soy phytoestrogen coumestrol against human breast cancer MCF-7 cells: Insights into the molecular mechanism. Food Chem. Toxicol. 2017, 99, 149-161. [CrossRef] [PubMed]

29. Zhou, Z.D.; Lim, T.M. Dopamine (DA) induced irreversible proteasome inhibition via DA derived quinones. Free Radic. Res. 2009, 43, 417-430. [CrossRef] [PubMed]

30. Adams, J. The proteasome: Structure, function, and role in the cell. Cancer Treat. Rev. 2003, 29, 3-9. [CrossRef]

31. Moore, B.S.; Eustáquio, A.S.; McGlinchey, R.P. Advances in and applications of proteasome inhibitors. Curr. Opin. Chem. Biol. 2008, 12, 434-440. [CrossRef] [PubMed]

32. Hochstrasser, M. Ubiquitin, proteasomes, and the regulation of intracellular protein degradation. Curr. Opin. Cell Biol. 1995, 7, 215-223. [CrossRef]

33. Bae, S.H.; Ryoo, H.M.; Kim, M.K.; Lee, K.H.; Sin, J.I.; Hyun, M.S. Effects of the proteasome inhibitor bortezomib alone and in combination with chemotherapeutic agents in gastric cancer cell lines. Oncol. Rep. 2008, 19, 1027-1032. [CrossRef] [PubMed]

34. Nam, S.; Smith, D.M.; Dou, P.D. Tannic Acid Potently Inhibits Tumor Cell Proteasome Activity, Increases p27 and Bax Expression, and Induces $\mathrm{G}_{1}$ Arrest and Apoptosis. Cancer Epidemiol. Biomark. Prev. 2001, 10, 1083-1088.

35. Yang, H.; Landis-Piwowar, K.R.; Chen, D.; Milacic, V.; Dou, Q.P. Natural Compounds with Proteasome Inhibitory Activity for Cancer Prevention and Treatment. Curr. Protein Pept. Sci. 2008, 9, 227-239. [CrossRef] [PubMed] 
36. Lee, J.; Peña, M.M.; Nose, Y.; Thiele, D.J. Biochemical characterization of the human copper transporter Ctr1. J. Biol. Chem. 2002, 277, 4380-4387. [CrossRef] [PubMed]

37. Nehybova, T.; Smarda, J.; Daniel, L.; Brezovsky, J.; Benes, P. Wedelolactone induces growth of breast cancer cells by stimulation of estrogen receptor signalling. J. Steroid. Biochem. Mol. Biol. 2015, 152, 76-83. [PubMed]

38. Jancekova, B.; Ondrouskova, E.; Knopfova, L.; Smarda, J.; Benes, P. Enzymatically active cathepsin D sensitizes breast carcinoma cells to TRAIL. Tumour Biol. 2016, 37, 10685-10696. [CrossRef] [PubMed]

39. Navratilova, J.; Hankeova, T.; Benes, P.; Smarda, J. Acidic pH of tumor microenvironment enhances cytotoxicity of the disulfiram/ $\mathrm{Cu}^{2+}$ complex to breast and colon cancer cells. Chemotherapy 2013, 59, 112-120. [CrossRef] [PubMed]

40. Pes, O.; Jungova, P.; Vyhnanek, R.; Vaculovic, T.; Kanicky, V.; Preisler, J. Off-line coupling of capillary electrophoresis to substrate-assisted laser desorption inductively coupled plasma mass spectrometry. Anal. Chem. 2008, 80, 8725-8732. [CrossRef] [PubMed]

41. Irwin, J.J.; Shoichet, B.K. ZINC-A free database of commercially available compounds for virtual screening. J. Chem. Inf. Model. 2005, 45, 177-182. [CrossRef] [PubMed]

42. Trott, O.; Olson, A.J. AutoDock Vina: Improving the speed and accuracy of docking with a new scoring function, efficient optimization, and multithreading. J. Comput. Chem. 2010, 31, 455-461. [CrossRef] [PubMed]

43. Sanner, M.F. Python: A programming language for software integration and development. J. Mol. Graph. Model. 1999, 17, 57-61. [PubMed]

44. Delano, W.T. The PyMol Molecular Graphics System, Version 1.5; Schrödinger, LLC: New York, NY, USA, 2009.

45. Durrant, J.; McCammon, J. NNScore 2.0: A Neural-Network Receptor-ligand Scoring Function. J. Chem. Inf. Model. 2011, 51, 2897-2903. [CrossRef] [PubMed]

(C) 2017 by the authors. Licensee MDPI, Basel, Switzerland. This article is an open access article distributed under the terms and conditions of the Creative Commons Attribution (CC BY) license (http:/ / creativecommons.org/licenses/by/4.0/). 